\title{
Work-Home Interference, Perceived Total Workload, and the Risk of Future Sickness Absence Due to Stress-Related Mental Diagnoses Among Women and Men: a Prospective Twin Study
}

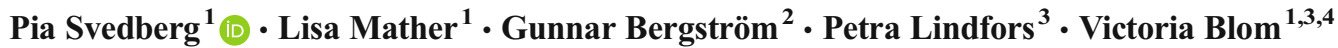

Published online: 21 June 2017

(C) The Author(s) 2017. This article is an open access publication

\begin{abstract}
Purpose Work-home interference has been proposed as an important explanation for sickness absence (SA). Previous studies show mixed results, have not accounted for familial factors (genetics and shared everyday environment), or investigated diagnosis specific SA. The aim was to study whether work-home interference and perceived total workload predict SA due to stress-related mental diagnoses, or SA due to other mental diagnoses, among women and men, when adjusting for various confounders and familial factors.

Methods This study included 11,916 twins, 19-47 years (49\% women). Data on work-to-home and home-to-work conflicts, perceived total workload, and relevant confounders were derived from a 2005 survey, and national register data on SA spells until 2013 were obtained. Odds ratios (ORs) with 95\% confidence intervals (CIs) were calculated. Discordant twin pair design was applied to adjust for familial factors.

Results Each one unit increase in work-to-home and home-towork conflicts, and perceived total workload was associated with higher odds for SA due to stress-related mental diagnoses
\end{abstract}

Pia Svedberg

Pia.Svedberg@ki.se

1 Division of Insurance Medicine, Department of Clinical Neuroscience, Karolinska Institutet, Berzeliusv. 3, 171 77 Stockholm, Sweden

2 Division of Intervention and Implementation Research, The Institute of Environmental Medicine, Karolinska Institutet, Stockholm, Sweden

3 Department of Psychology, Stockholm University, Stockholm, Sweden

4 The Swedish School of Sport and Health Sciences, Stockholm, Sweden and to SA due to other mental diagnoses among women, when adjusting for sociodemographic factors (ORs 1.15-1.31). Including health or familial factors, no associations remained. For men, each one unit increase in work-to-home conflicts was associated with higher odds for SA due to stress-related diagnoses (ORs 1.23-1.35), independently of confounders. Conclusion Work-to-home conflict was independently associated with future SA due to stress-related diagnoses among men only. Health- and work-related factors seem to be important confounders when researching work-home interference, perceived total workload, and SA. Not including such confounders involves risking drawing incorrect conclusions. Further studies are needed to confirm sex differences and whether genetic factors are important for the associations studied.

Keywords Sick leave · Work-home interference $\cdot$ Work disability $\cdot$ Twins $\cdot$ Work load $\cdot$ Gender

\section{Background}

Changes in work and home domains involve more people struggling to combine work and family life. Unequal distribution of home duties along with a high total workload has been suggested to explain why women tend to report negative work-home balance to a higher degree than men $[1,2]$. However, an imbalance between work and home responsibilities has been associated with sub-optimal health in both women and men [2-5]. Considering this, the interference between work and family life has been suggested as an important explanation for sickness absence (SA) [2, 6-10], alongside factors relating to individuals' health, work environment, sociodemographic, and lifestyle factors [11]. 
Despite an increased focus on SA, knowledge of specific risk factors, including work-home interference, remains inconsistent. Most research focuses on overall SA, while fewer studies include SA due to specific diagnoses. Currently, mental disorders that include the ICD-10 diagnosis F43, i.e., reaction to severe stress and adjustment disorders (from now referred to as stress-related mental disorders), are the most common reasons for SA, especially among women and younger individuals in Sweden and in other European countries [12, 13]. However, many studies of SA risk factors are hampered by cross-sectional designs or selection biases relating to health factors or family background. Various health conditions including mental disorders are influenced by genetic factors, which in turn may influence the risk of experiencing stress. Further, previous studies have shown that SA is moderately heritable. But, a population-based twin-setting including twins sharing their genes and having grown up in the same family allows controlling for genetic and shared environmental (familial) confounders. No previous study has used this design to investigate work-home interference as a risk factor for SA.

Negative health outcomes of work-home interference may result of negative spillover effects due to situations including an inter-role conflict, i.e., being involved at work may put strain on the family role, or vice versa $[14,15]$. Consequently, two types of work-home interference may follow: work-to-home conflict referring to work-role demands having an unfavorable impact on the home and family roles and home-to-work conflict which refers to demands at home having an unfavorable impact on individuals' work roles [16, 17]. Many studies report spillover effects but also find that dispositional factors and work characteristics are important for work-home interference [18-20].

Regarding SA, studies are few and findings mixed. In one study, work-to-home conflict was associated with almost threefold higher odds of SA among men in higher socioeconomic strata, while no such association emerged for women [8]. Another study found that women reporting high work-tohome conflict were at higher risk for SA [6]. Also, recent research found that gender, age, and family situation, including having children, play a role for the associations [6, 21]. Others have found that home-to-work conflict is associated with long SA duration (>10 SA days) and high SA frequency in both women and men, also when adjusting for sociodemographic factors, health indicators, and psychosocial factors [7]. Jansen and colleagues [9] found a clear association between home-to-work conflicts but not for work-to-home conflict and SA.

With previous studies showing that work-home interference is associated with sub-optimal health, mental disorders, and burnout $[4,5,22,23]$, it seems reasonable to assume that work-home interference is a risk factor for SA due to stressrelated mental diagnoses or other mental diagnoses. However, with previous research on work-home interference having focused on the association to SA in general, using crosssectional or prospective designs with shorter follow-ups, it is unclear whether effects differ between SA diagnoses when using a follow-up time of several years.

So far, there are no twin studies of work-home interference or the perception of total workload and the risk of future SA. But, a recent twin study showed that both work-to-home and home-to-work conflicts were associated with burnout and that genetic factors seemed to confound the association between home-to-work conflict and burnout [4]. Another study identified high job demands and job strain as risk factors for SA due to mental disorders, with familial factors seeming important for the association between job support and incident SA [24]. Moreover, recent research indicates that childhood experiences of the family influence how adults perceive work strain and demands [25]. Furthermore, twin studies have shown the importance of genetics for SA [26, 27] but also for other individual factors. This includes associations with abilities relevant for handling work-home balance and experiences of a high workload, such as coping behaviors [28] and cognitive resources $[29,30]$. Additionally, a longitudinal study found that work-home interference is fairly stable throughout life and not only limited to the early working career [31]. This suggests that some dispositional factors, such as personality, which is highly influenced by genetics, could be involved. Moreover, it is well-known that genetics play a role in mental disorders [32] underlying work disability even though one twin study has suggested that the association between internalizing mental disorders and SA is influenced by unique environmental factors rather than by genetics [33]. But, taking together previous findings makes it reasonable to assume that the associations between work-home interference and SA are influenced by familial factors in addition to psychosocial, health, or work-related factors.

The aim of the present prospective study was to investigate whether work-to-home and home-to-work conflicts and perceived total workload are risk factors for future SA due to stress-related mental disorders (ICD-10, diagnosis F43), and SA due to other mental disorders, among women and men, also when adjusting for confounders including familial (genetics and shared environment) factors.

\section{Methods}

\section{Study Population}

The source population consisted of 25,496 twins born between 1959 and 1985 of the Swedish Twin project of Disability pension and Sickness absence (STODS) who participated in the Study of Twin Adults: Genes and Environment (STAGE) web-based survey conducted by the Swedish Twin Registry in 2005 [34]. The present study investigated work- 
home interference and perceived total workload in association to SA. So, only data from working individuals were included. Individuals being disability pensioned at the time of interview or only having SA spells due to non-mental diagnoses at follow-up were excluded. The final study sample included 11,916 twins ( $49 \%$ women), aged $19-47$ (mean $=35.4, S D$ 6.8) (see Fig. 1 for inclusion criteria). Of these, 2385 were complete pairs, 942 monozygotic (MZ) pairs, 723 same-sex dizygotic (DZ) pairs, and 720 opposite-sex pairs. Also 7146 single twins were included, i.e., the twin sibling did not respond to STAGE, or belonged to pairs of unknown zygosity, or were excluded based on the above criteria. For details on zygosity determination in the Swedish Twin Registry, see Lichtenstein et al. [34].

\section{Outcomes and Follow-Up Time}

SA data were obtained from the National Social Insurance Agency MicroData for Analyses of Social insurance database (MiDAS) and linked to each individual using the Swedish tendigit personal identification number. All individuals in Sweden above the age of 16, with an income from work or unemployment benefits, can receive sickness benefits paid by the Social Insurance Agency when disease or injury has caused reduced work capacity. Employees receive sick pay from their employers during the first 14 days after a qualifying day (usually more qualifying days for self-employed) without benefits. Diagnosis-specific SA during follow-up was defined based on ICD-10 codes [35]. SA was operationalized as having at least one incident spell lasting longer than 14 days during follow-up i.e., between the date of STAGE survey response (varying between $11 / 01 / 2004$ to $04 / 21 / 2006$ ) and $12 /$ $31 / 2013$. Two outcome variables were created; $S A$ due to stress-related diagnoses (ICD-10 F43) and SA due to other mental diagnoses (other diagnoses in the F-chapter, except for F43 episodes during follow-up). Hierarchy was applied and priority was given to spells in stress-related mental diagnoses, followed by other mental diagnoses. No SA spell during follow-up was used as reference.

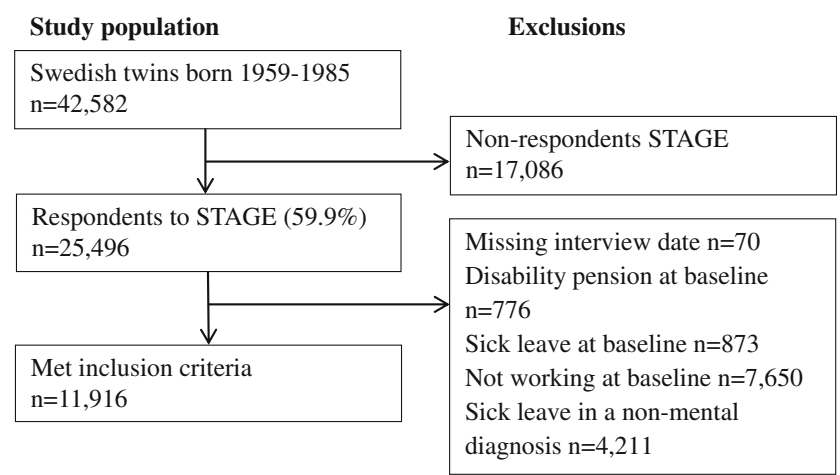

Fig. 1 Flowchart of the study sample: inclusion and exclusion criteria

\section{Exposures}

Work-home interference was measured with the following two questions: "Do the demands in your work affect your home and family life in a negative way" (work-to-home conflict) and "Do the demands in your home/family affect your work in a negative way" (home-to-work conflict). These items were originally developed for the General Nordic Questionnaire for psychological and social factors at work (QPSNordic) [36].

Perceived total workload was assessed using the question "Do you have difficulties getting sufficient time for both work and personal life?" It measures an individual's perception of the total workload, which is distinct from the actual amount of work.

For all three variables, the STAGE used a four-point response format: $1=$ always, $2=$ sometimes, $3=$ almost never, and $4=$ never. Responses were reversed with high scores indicating more conflict or a higher perceived workload.

\section{Confounders}

This study includes factors previously associated with workhome interference and SA.

\section{Sociodemographic Factors}

Age was included as a continuous variable derived by subtracting the date of response to STAGE from the birthdate. Sex was dichotomous (women and men). The highest level of education was categorized into three groups (1) elementary school, (2) vocational school, and (3) university degree (military school and vocational university were included in category 3 and residential college for adult education in category 2). Marital status was grouped into married/cohabiting or not. Living with children was measured as a dichotomous variable, stating whether an individual had children living at home or not.

\section{Work- and Health-Related Factors}

Work status was measured as a dichotomous variable with an individual reporting working full-time, being full-time employed, or full-time self-employed, or a combination of part-time employment and part-time self-employment (working full-time), and others i.e., not working full-time. The Swedish translation [37] of the Karasek and Theorell [38] questionnaire was used to assess job demands, control, and support. Responses were given on a four-point Likert scale, from $1=$ do not agree to $4=$ agree entirely. Mean scores were calculated of job demands, control, and support and used as continuous variables. Self-rated health (SRH) was asked for in STAGE with the question "How would you rate your general health status?" with response alternatives excellent, good, 
moderate, fairly poor, and poor. With few responses in the lowest categories, 'fairly poor' and 'poor' were collapsed into one category. Previous sick leave was based on MiDAS data (episodes of SA > 14 days in a row) between 2003 and STAGE response (approximately a 2 -year period) (yes/no).

\section{Statistical Analyses}

Logistic regression analyses were used to assess odds ratios (ORs) with $95 \%$ confidence intervals (CIs), stratified by sex to assess the associations between work-to-home conflict, home-to-work conflict, perceived total workload, and SA. The responses "do not know/do not want to answer" were treated as missing values. Analyses were adjusted for the study sample including twin pairs rather than independent individuals by using the clustered robust standard error. In the analysis of the whole sample, covariates were entered in three blocks: first sociodemographic factors (age, education, and marital status) were entered (model 1), then living with children, work status, job demands, control, and support were entered (model 2), and finally previous history of SA and SRH were added (model 3). An additional analysis combining women and men, adjusting for age and sex, was also conducted, and we tested the interaction effects with sex. Co-twin control (conditional logistic regression) analyses based on same-sex discordant $\mathrm{MZ}$ and $\mathrm{DZ}$ twin pairs were conducted to adjust for familial (genetics and shared family environment) confounding $[39,40]$. A twin pair was treated as discordant if only one twin of a pair had incident SA during follow-up. In co-twin control analyses, twins in a pair are optimally matched on genetics (MZ $100 \%$ and DZ on average $50 \%$ ) and shared environmental factors $(100 \%)$ when reared together, and for age and sex. An influence of familial factors is indicated if an association found in the whole sample disappears or changes considerably in the analyses of discordant twin pairs. If the association is stronger in DZ than $\mathrm{MZ}$ pairs, genetics rather than shared environmental factors are of importance, while familial factors will be assumed to play a minor role if the association is found in the analyses of both the whole sample and of discordant twin pairs. Co-twin analyses were conducted both stratified by sex (MZ and DZ pairs combined) and stratified by zygosity. In addition, an unconditional logistic regression analysis of all the 418 individuals belonging to SA discordant twin pairs was conducted. All analyses were conducted using STATA IC 12.1.

\section{Results}

Table 1 presents descriptive statistics for the whole sample ( $n=11,916)$, by sex and SA status during follow-up. More women than men had SA spells during follow-up. Among women, SA due to stress-related mental diagnoses was more common than SA due to other mental diagnoses. Among men, SA due to stress-related and SA due to other mental diagnoses were equally common. Table 2 shows results of the logistic regression analyses stratified by sex, which revealed that for women, each one unit increase in work-to-home and home-towork conflicts and perceived total workload were associated with higher odds for future SA due to stress-related mental diagnoses and to SA due to other mental diagnoses, also when adjusting for sociodemographic factors (ORs 1.15-1.31). The associations between work-to-home conflict, perceived total workload, and SA due to stress-related mental diagnoses were non-significant after adjusting for work-related factors and living with children. Similar results emerged for the associations between home-to-work conflict, perceived total workload, and SA due to other mental diagnoses. When adjusting for previous SA history and SRH, no associations remained.

For men, each one-unit increase in work-to-home conflicts was associated with higher odds for SA due to stress-related diagnoses, also after adjusting for all covariates (ORs in models $1-3 ; 1.23-1.35$ ). Only the crude model showed an association between home-to-work conflicts and SA due to stress-related diagnoses for men. No associations emerged between the exposures and SA due to other mental disorders.

Analyzing women and men together, adjusting for age and sex, each one unit increase in all three exposures were significantly associated with higher odds (ORs 1.12-1.28) for future SA due to stress-related or other mental diagnoses (see Table 3). We found no statistically significant interaction effects with sex.

Discordant twin pair analyses (see Table 3) showed no statistically significant associations between work-to-home or home-to-work conflicts or perceived total workload and $\mathrm{SA}$ due to stress-related or other mental diagnoses for MZ or DZ twin pairs. However, for discordant DZ twin pairs estimates followed those of the whole sample but with less precision. This suggests that genetic factors may be of importance for the studied associations. Results of the unconditional analysis of the 418 individuals belonging to SA discordant pairs showed non-significant estimates for the associations between work-to-home and home-to-work conflicts, perceived total workload, and SA due to stress-related or other mental diagnoses. Estimates were as expected in between the estimates of $\mathrm{DZ}$ and MZ pairs (conditional models) and with less precision than estimates of the whole cohort (Table 3). For comparative purposes, Table 2 presents ORs for MZ and DZ discordant twin pairs combined but stratified by sex; none of the estimates reached statistical significance.

\section{Discussion}

This prospective twin cohort study of 11,916 working-age women and men provided a unique opportunity to investigate workhome interference and perceived total workload as risk factors for SA due to stress-related or due to other mental diagnoses. We took advantage of a discordant twin pair design to account for familial 
Table 1 Frequencies of exposures and covariates for 11,916 Swedish twin individuals stratified by sickness absence (SA) status during follow-up and sex

\begin{tabular}{|c|c|c|c|c|c|c|}
\hline & \multicolumn{2}{|l|}{ No SA } & \multicolumn{2}{|c|}{ SA due to stress-related mental diagnoses } & \multicolumn{2}{|c|}{ SA due to other mental diagnoses } \\
\hline & Women $(n=4707)$ & $\operatorname{Men}(n=5697)$ & Women $(n=656)$ & $\operatorname{Men}(n=197)$ & Women $(n=459)$ & $\operatorname{Men}(n=200)$ \\
\hline Exposures & $n(\%) /$ mean $(\mathrm{SD})$ & $n(\%) /$ mean $(\mathrm{SD})$ & $n(\%) /$ mean $(\mathrm{SD})$ & $n(\%) /$ mean $(\mathrm{SD})$ & $n(\%) /$ mean $(\mathrm{SD})$ & $n(\%) /$ mean $(\mathrm{SD})$ \\
\hline \multicolumn{7}{|c|}{ Work-to-home conflict (WHC) } \\
\hline 1. never/almost never & $1594(33.9)$ & $2060(36.2)$ & $164(25.0)$ & $53(26.9)$ & $132(28.8)$ & $68(34.0)$ \\
\hline 2. Seldom & $985(20.9)$ & $1294(22.7)$ & $132(20.1)$ & $36(18.3)$ & 86 (18.7) & $43(21.5)$ \\
\hline 3. Sometimes & $1462(31.0)$ & $1701(29.8)$ & $249(38.0)$ & $78(39.6)$ & $155(33.8)$ & $59(29.5)$ \\
\hline 4. Often & $243(5.2)$ & $336(5.9)$ & $48(7.3)$ & $18(9.1)$ & $55(12.0)$ & $17(8.5)$ \\
\hline Missing & $423(9.0)$ & $306(5.4)$ & $63(9.6)$ & $12(6.1)$ & $31(6.7)$ & $13(6.5)$ \\
\hline WHC (mean, 1-4) & $2.1(1.0)$ & $2.1(1.0)$ & $2.3(1.0)$ & $2.3(1.0)$ & $2.3(1.0)$ & $2.1(1.0)$ \\
\hline \multicolumn{7}{|c|}{ Home-to-work conflict (HWC) } \\
\hline 1. Never/almost never & 2476 (52.6) & $3041(53.4)$ & $292(44.5)$ & $91(46.2)$ & $235(51.2)$ & $109(54.5)$ \\
\hline 2. Seldom & $1119(23.8)$ & $1411(24.8)$ & $164(25.0)$ & $52(26.4)$ & $97(21.1)$ & $40(20.0)$ \\
\hline 3. Sometimes & $634(13.5)$ & $865(15.2)$ & $121(18.5)$ & $40(20.3)$ & $82(17.9)$ & $35(17.5)$ \\
\hline 4. Often & $53(1.1)$ & $71(1.2)$ & $16(2.4)$ & $3(1.5)$ & $14(3.0)$ & $4(2.0)$ \\
\hline Missing & $425(9.0)$ & $309(5.4)$ & $63(9.6)$ & $11(5.6)$ & $31(6.8)$ & $12(6.0)$ \\
\hline HWC (mean, 1-4) & $1.6(0.8)$ & $1.6(0.8)$ & $1.8(0.9)$ & $1.8(0.8)$ & $1.7(0.9)$ & $1.7(0.9)$ \\
\hline \multicolumn{7}{|c|}{ Perceived total workload (PTW) } \\
\hline 1. Never/almost never & $917(19.5)$ & $1236(21.7)$ & $102(15.5)$ & $39(19.8)$ & $87(19.0)$ & $43(21.5)$ \\
\hline 2. Seldom & $697(14.8)$ & $966(17.0)$ & $78(11.9)$ & $28(14.2)$ & $47(10.2)$ & $31(15.5)$ \\
\hline 3. Sometimes & $1758(37.4)$ & $2014(35.3)$ & $241(36.7)$ & $60(30.5)$ & $169(36.8)$ & $66(33.0)$ \\
\hline 4. Often & $919(19.5)$ & $1181(20.7)$ & $171(26.1)$ & $59(29.9)$ & $126(27.5)$ & $47(23.5)$ \\
\hline Missing & $416(8.8)$ & $300(5.3)$ & $64(9.8)$ & $11(5.6)$ & $30(6.5)$ & $13(6.5)$ \\
\hline PTW (mean, 1-4) & $2.6(1.0)$ & $2.6(1.1)$ & $2.8(1)$ & $2.8(1.1)$ & $2.8(1.1)$ & $2.6(1.1)$ \\
\hline Covariates & $n(\%) /$ mean $(\mathrm{SD})$ & $n(\%) /$ mean $(\mathrm{SD})$ & $n(\%) /$ mean $(\mathrm{SD})$ & $n(\%) /$ mean $(\mathrm{SD})$ & $n(\%) /$ mean $(\mathrm{SD})$ & $n(\%) /$ mean $(\mathrm{SD})$ \\
\hline Age (range 19-47) & $35.6(6.9)$ & $35.1(6.8)$ & $35.9(6.4)$ & $36.1(6.7)$ & $34.9(7.1)$ & $36.1(6.9)$ \\
\hline \multicolumn{7}{|l|}{ Education } \\
\hline Elementary/vocational & $1955(41.5)$ & $2814(49.4)$ & $277(42.2)$ & $97(49.2)$ & $228(49.7)$ & $111(55.5)$ \\
\hline University & $2475(52.6)$ & $2629(46.1)$ & $325(49.6)$ & $87(44.2)$ & $187(40.7)$ & $78(39.0)$ \\
\hline Missing & $277(5.9)$ & $254(4.5)$ & $54(8.2)$ & $13(6.6)$ & $44(9.6)$ & $11(5.5)$ \\
\hline \multicolumn{7}{|l|}{ Marital status } \\
\hline Married/cohabiting & $3426(72.8)$ & $3966(69.6)$ & $491(74.8)$ & $135(68.5)$ & $319(69.5)$ & $124(62.0)$ \\
\hline Other & $1273(27.0)$ & $1726(30.3)$ & $165(25.2)$ & $61(31.0)$ & $140(30.5)$ & $76(38.0)$ \\
\hline Missing & $8(0.2)$ & $5(0.1)$ & 0 & $1(0.5)$ & 0 & 0 \\
\hline \multicolumn{7}{|l|}{ Living with children } \\
\hline Yes & $2811(59.7)$ & $2806(49.3)$ & $427(65.1)$ & $95(48.2)$ & $274(59.7)$ & $100(50.0)$ \\
\hline No & $1896(40.3)$ & $2891(50.7)$ & 229 (34.9) & $102(51.8)$ & $185(40.3)$ & $100(50.0)$ \\
\hline \multicolumn{7}{|l|}{ Work full time } \\
\hline Yes & $3393(72.1)$ & $5378(94.4)$ & 488 (74.4) & 189 (95.9) & $322(70.2)$ & $184(92.0)$ \\
\hline No & $1314(27.9)$ & $319(5.6)$ & $168(25.6)$ & $8(4.1)$ & $137(29.8)$ & $16(8.0)$ \\
\hline Job demands (mean, 1-4) & $2.5(0.6)$ & $2.5(0.6)$ & $2.7(0.6)$ & $2.6(0.6)$ & $2.6(0.6)$ & $2.6(0.6)$ \\
\hline Control (mean, 1-4) & $3(0.6)$ & $3.1(0.5)$ & $3(0.6)$ & $3.1(0.6)$ & $2.9(0.6)$ & $3.1(0.6)$ \\
\hline Support (mean, 1-4) & $3.4(0.5)$ & $3.4(0.5)$ & $3.3(0.5)$ & $3.3(0.5)$ & $3.3(0.5)$ & $3.3(0.5)$ \\
\hline \multicolumn{7}{|l|}{ Previous sick leave } \\
\hline Yes & $669(14.2)$ & $409(7.2)$ & 229 (34.9) & $37(18.8)$ & $183(39.9)$ & $51(25.5)$ \\
\hline No & $4038(85.8)$ & $5288(92.8)$ & $427(65.1)$ & $160(81.2)$ & $276(60.1)$ & $149(74.5)$ \\
\hline \multicolumn{7}{|l|}{ Self-rated health } \\
\hline Excellent & $1639(34.8)$ & $2038(35.8)$ & $158(24.1)$ & $52(26.4)$ & $83(18.1)$ & $48(24.0)$ \\
\hline Good & $2342(49.8)$ & $2762(48.5)$ & $333(50.7)$ & $106(53.8)$ & $217(47.3)$ & $84(42.0)$ \\
\hline Moderate & $567(12.0)$ & $672(11.8)$ & 137 (20.9) & $31(15.7)$ & $112(24.4)$ & $43(21.5)$ \\
\hline Fairly poor/poor & $78(1.7)$ & $55(0.9)$ & $15(2.3)$ & $5(2.6)$ & $32(7.0)$ & $16(8.0)$ \\
\hline Missing & $81(1.7)$ & $170(3.0)$ & $13(2.0)$ & $3(1.5)$ & $15(3.2)$ & $9(4.5)$ \\
\hline
\end{tabular}

(genetics and shared environment) confounding. The associations were also studied while adjusting for several relevant confounders. Specifically, we found no significant associations between work-home interference, perceived total workload, and SA due to stress-related or other mental diagnoses for women when adjusting for various confounders. However, among men, workto-home conflict was associated with SA due to stress-related mental diagnoses, independently of the confounding factors. In line with expectations, various confounders including work or health aspects and perhaps genetics seemed to explain most of the associations.

Even though differences in employment rates between women and men have decreased in many countries, traditional gender patterns regarding, for example, responsibility for the household and children remain. Moreover, the labor market is gendered, as is work disability. This means that the potential sex differences 
Table 2 Odds ratios (OR) with 95\% confidence intervals (CI) for work-home interference and perceived total workload as predictors for sickness absence (SA) due to stress-related diagnoses and SA due to other mental diagnoses among 11,916 Swedish twins and discordant twin pair analysis (co-twin) among same-sex monozygotic (MZ) and dizygotic (DZ) twin pairs (116 discordant for SA due to stressrelated and 93 discordant for SA due to other mental diagnoses). Analyses stratified by sex

\begin{tabular}{|c|c|c|c|c|}
\hline & \multicolumn{2}{|c|}{ SA due to stress-related diagnoses } & \multicolumn{2}{|c|}{ SA due to other mental disorders } \\
\hline & OR & $95 \% \mathrm{CI}$ & OR & $95 \% \mathrm{CI}$ \\
\hline \multicolumn{5}{|l|}{ Women } \\
\hline \multicolumn{5}{|l|}{ Work-to-home conflict } \\
\hline Crude & 1.27 & $1.16-1.38$ & 1.27 & $1.14-1.41$ \\
\hline Model 1 & 1.26 & $1.14-1.38$ & 1.31 & $1.17-1.47$ \\
\hline Model 2 & 1.12 & $1.00-1.24$ & 1.28 & $1.12-1.46$ \\
\hline Model 3 & 1.07 & $0.95-1.19$ & 1.15 & $1.00-1.32$ \\
\hline Co-twin model (MZ + DZ) & 1.17 & $0.77-1.78$ & 1.03 & $0.66-1.59$ \\
\hline \multicolumn{5}{|l|}{ Home-to-work conflict } \\
\hline Crude & 1.29 & $1.16-1.43$ & 1.19 & $1.05-1.35$ \\
\hline Model 1 & 1.28 & $1.14-1.42$ & 1.20 & $1.05-1.38$ \\
\hline Model 2 & 1.18 & $1.04-1.32$ & 1.15 & $0.99-1.33$ \\
\hline Model 3 & 1.12 & $0.99-1.27$ & 1.05 & $0.90-1.22$ \\
\hline Co-twin model (MZ + DZ) & 0.92 & $0.65-1.31$ & 1.05 & $0.67-1.64$ \\
\hline \multicolumn{5}{|l|}{ Perceived total workload } \\
\hline Crude & 1.19 & $1.09-1.30$ & 1.15 & $1.04-1.28$ \\
\hline Model 1 & 1.20 & $1.09-1.31$ & 1.17 & $1.06-1.30$ \\
\hline Model 2 & 1.07 & $0.97-1.19$ & 1.12 & $0.99-1.26$ \\
\hline Model 3 & 1.03 & $0.93-1.15$ & 1.03 & $0.91-1.16$ \\
\hline Co-twin model (MZ + DZ) & 1.20 & $0.86-1.67$ & 0.84 & $0.55-1.27$ \\
\hline \multicolumn{5}{|l|}{ Men } \\
\hline \multicolumn{5}{|l|}{ Work-to-home conflict } \\
\hline Crude & 1.32 & $1.14-1.54$ & 1.08 & $0.93-1.26$ \\
\hline Model 1 & 1.35 & $1.15-1.57$ & 1.09 & $0.92-1.28$ \\
\hline Model 2 & 1.25 & $1.05-1.49$ & 1.05 & $0.87-1.26$ \\
\hline Model 3 & 1.23 & $1.03-1.47$ & 0.93 & $0.77-1.12$ \\
\hline Co-twin model (MZ + DZ) & 1.54 & $0.91-2.61$ & 0.78 & $0.39-1.55$ \\
\hline \multicolumn{5}{|l|}{ Home-to-work conflict } \\
\hline Crude & 1.22 & $1.03-1.45$ & 1.04 & $0.86-1.26$ \\
\hline Model 1 & 1.20 & $1.00-1.45$ & 1.04 & $0.85-1.28$ \\
\hline Model 2 & 1.13 & $0.92-1.39$ & 1.02 & $0.82-1.27$ \\
\hline Model 3 & 1.08 & $0.88-1.33$ & 0.91 & $0.72-1.15$ \\
\hline Co-twin model (MZ + DZ) & 1.18 & $0.65-2.17$ & 0.85 & $0.48-1.49$ \\
\hline \multicolumn{5}{|l|}{ Perceived total workload } \\
\hline Crude & 1.16 & $1.00-1.35$ & 1.04 & $0.90-1.20$ \\
\hline Model 1 & 1.16 & $0.99-1.36$ & 1.04 & $0.90-1.21$ \\
\hline Model 2 & 1.08 & $0.92-1.28$ & 1.01 & $0.85-1.20$ \\
\hline Model 3 & 1.07 & $0.91-1.27$ & 0.98 & $0.83-1.17$ \\
\hline Co-twin model (MZ + DZ) & 1.03 & $0.65-1.61$ & 1.05 & $0.68-1.62$ \\
\hline
\end{tabular}

Statistically significant ORs in italics. Model 1: adjusted for age, education, and marital status; model 2: adjusted for age, education, marital status, living with children, work full time, job demands, control, and support; model 3: adjusted for age, education, marital status, living with children, work full time, job demands, control, support, previous sick leave, and self-rated health.

$M Z$ monozygotic, $D Z$ dizygotic found in the present study may reflect an unequal division of home responsibilities and/or unequal opportunities in working life. However, these results need to be replicated in studies including larger samples before drawing any firm conclusions regarding possible sex differences. Previous research suggests that the unequal sharing of household responsibilities is associated with negative health outcomes, especially among women [2]. Our results suggest that, for women, the associations between work-to-home 
Table 3 Odds ratios (OR) with 95\% confidence intervals (CI) for work-home interference and perceived total workload as predictors for sickness absence (SA) due to stress-related or other mental diagnoses; for the whole sample (11,916 twins) and of the discordant (co-twin) same-sex twin pairs (116 discordant for SA due to stress-related and 93 discordant for SA due to other mental diagnoses) by zygosity group

\begin{tabular}{|c|c|c|c|c|}
\hline & \multirow{2}{*}{$\begin{array}{l}\text { Whole sample } \\
\text { OR }(95 \% \mathrm{CI})\end{array}$} & \multicolumn{3}{|l|}{ Co-twin analysis } \\
\hline & & Discordant twin pairs ${ }^{\mathrm{b}}$ OR $(95 \% \mathrm{CI})$ & DZ OR $(95 \% \mathrm{CI})$ & $\mathrm{MZ}$ OR $(95 \% \mathrm{CI})$ \\
\hline \multicolumn{5}{|c|}{ SA due to stress-related mental diagnoses } \\
\hline Work-to-home conflict & $1.28(1.18-1.38)$ & $1.22(0.96-1.56)$ & $1.47(0.95-2.28)$ & $1.13(0.69-1.84)$ \\
\hline Home-to-work conflict & $1.26(1.15-1.38)$ & $0.99(0.76-1.29)$ & $1.31(0.83-2.06)$ & $0.72(0.47-1.11)$ \\
\hline Perceived total workload & $1.18(1.09-1.27)$ & $1.10(0.88-1.37)$ & $1.29(0.88-1.89)$ & $1.00(0.69-1.46)$ \\
\hline \multicolumn{5}{|c|}{ SA due to other mental diagnoses } \\
\hline Work-to-home conflict & $1.21(1.11-1.33)$ & $0.97(0.76-1.22)$ & $1.20(0.73-1.96)$ & $0.68(0.38-1.21)$ \\
\hline Home-to-work conflict & $1.15(1.03-1.28)$ & $1.00(0.70-1.42)$ & $1.22(0.73-2.04)$ & $0.79(0.49-1.26)$ \\
\hline Perceived total workload & $1.12(1.03-1.22)$ & $0.95(0.74-1.21)$ & $1.05(0.73-1.52)$ & $0.73(0.44-1.21)$ \\
\hline
\end{tabular}

Statistically significant ORs in italics

$M Z$ monozygotic, $D Z$ dizygotic

${ }^{a}$ Women and men combined, adjusted for sex and age

${ }^{\mathrm{b}}$ Unconditional logistic regression analysis of all the 232 and 186 individuals, respectively, belonging to SA discordant twin pairs

and home-to-work conflicts, perceived total workload, and SA due to stress-related or due to other mental diagnoses were similar. Also, these associations were explained either by work- or healthrelated factors, and potentially genetics with these results being less clear. For men, only work-to-home conflict was associated to SA due to stress-related mental diagnoses and independently of the confounders. This follows previous research showing sex differences in the association between work-to-home conflict and SA in general [8]. Thus, the results indicate that the gendered work and family life may manifest differently for women and men in relation to SA. For men, this involves excessive demands at work, and for women, it is most likely the balancing of excessive demands both at home and at work. But for women, the importance of ill-health and/or genetic vulnerability for diseases or factors such as e.g. neuroticism and conscientiousness [41] is pronounced. Many studies show that genetic factors are important for mental disorders [32]. Women are more often diagnosed with mental disorders and consequently also on sick leave due to such disorders to a higher degree then men [12]. Most likely, the genetic influences in the present study reflect health factors, since the previous history of SA and SRH confounds the results of associations in the whole cohort. More research, with larger samples, is needed to identify explanatory factors for the association between work-to-home conflict and SA due to stress-related mental diagnoses among men.

\section{Strengths and Limitations}

This study has several strengths including the large and genetically informed population-based sample, objective SA data of high quality with complete coverage from a national register, and a prospective cohort design. Also, extensive survey data including validated measures of work-home interference and relevant confounders were available. Using single-item exposures may introduce measurement error. However, the items included in the present analyses were originally developed for the General Nordic Questionnaire for Psychological and Social factors at work (QPSNordic) and the psychometric testing of this questionnaire suggests its good qualities for assessing health-related factors at work [36], and the items have been widely used in studies of work-home interference and various outcomes (e.g., [8]) and are in accordance with those of Frone and colleagues [17]. A unique strength includes the possibility to control for familial confounding using the discordant twin pairs i.e., to determine whether an association is likely to reflect a causal relationship [39]. Here, we found some support for a direct effect of work-to-home conflict on future SA due to stress-related mental diagnoses but only among men. However, any interpretation of the results needs to acknowledge study limitations. First, questionnaire data always include some missing data. Yet, in the final sample, the amount of missing data based on self-report measures and included confounders were low. Second, without survey follow-ups, exposures were only assessed at a single time-point. Consequently, it is unclear whether reports of work-home interference or confounding factors change, and if such changes influence the risk of SA. Further, only twins, aged 19-47, born in Sweden were included which reduces generalizability to other groups such as immigrants, other countries, and older adults. Also, the issue of whether physicians are able to reliably distinguish between F43 and other ICD diagnoses of mental disorders should be considered. For this study, only one main SA diagnosis was available meaning that misclassification or comorbidity may be present. Recent findings also show changes in the primary diagnosis to a diagnosis from another diagnostic chapter during the same episode; this happened to $7.1 \%$ of women cases and $6.6 \%$ of men cases. However, such a change of the primary diagnosis was least common among those 
initially sick-listed for mental and musculoskeletal disorders [42]. The patterns of diagnostic changes were similar for women and men. Yet, changes within a diagnosis chapter remain to be investigated. Finally, we cannot rule out the influence of familial factors on the associations studied; estimates of the discordant twin pair analyses had lower precision and need to be interpreted with caution. Additional studies with larger samples are needed to confirm or reject the influence of genetic factors on the association between work-home interference and SA.

\section{Conclusions}

This study suggests potential sex differences in the associations between work-home interference, perceived total workload, and SA due to stress-related and other mental diagnoses, also with respect to influential factors. For men, an independent association emerged between work-to-home conflict and SA due to stress-related mental diagnoses. Work, health, and also potentially genetic factors seem to be important confounders, particularly among women. Importantly, disregarding health status and work factors may result in erroneous conclusions regarding the true effect of work-home interference on future SA.

Acknowledgments This study was financially supported by AFA Insurance (Dnr 140246). STODS has been supported by the Swedish Research Council (521-2008-3054), the Swedish Research Council for Health, Working Life and Welfare (2007-0830), and the Swedish Society of Medicine. The Swedish Twin Registry was supported by the Department of Higher Education and the Swedish Research Council. STAGE was supported by the National Institute of Health, USA (grants DK 066134 and CA 085739).

Contributors VB and PS originated the idea. LM analyzed the data in consultation with PS, VB, GB, and PL. PS wrote the first and subsequent drafts of the manuscript, with important intellectual input from all the coauthors. All authors contributed in designing the study and to the interpretation of the results and to the writing and approval of the final article.

\section{Compliance with Ethical Standards}

Funding This study was financially supported by AFA Insurance (Dnr 140246). STODS has been supported by the Swedish Research Council (521-2008-3054), the Swedish Research Council for Health, Working Life and Welfare (2007-0830), and the Swedish Society of Medicine. The Swedish Twin Registry was supported by the Department of Higher Education and the Swedish Research Council. STAGE was supported by the National Institute of Health, USA (grants DK 066134 and CA 085739).

Competing Interests The authors declare that they have no conflict of interest.

Ethics Approval All procedures performed in studies involving human participants were in accordance with the ethical standards of the institutional and/or national research committee and with the 1964 Helsinki declaration and its later amendments or comparable ethical standards. This study was approved by the Regional Ethical Review Board in Stockholm, Sweden.
Open Access This article is distributed under the terms of the Creative Commons Attribution 4.0 International License (http:// creativecommons.org/licenses/by/4.0/), which permits unrestricted use, distribution, and reproduction in any medium, provided you give appropriate credit to the original author(s) and the source, provide a link to the Creative Commons license, and indicate if changes were made.

\section{References}

1. Lundberg U. Stress hormones in health and illness: the roles of work and gender. Psychoneuroendocrinology. 2005;30(10):101721.

2. Eek F, Axmon A. Gender inequality at home is associated with poorer health for women. Scand J Public Health. 2015;43(2):17682.

3. Staland-Nyman C, Alexanderson K, Hensing G. Associations between strain in domestic work and self-rated health: a study of employed women in Sweden. Scand J Public Health. 2008;36(1): 21-7.

4. Blom V, Sverke M, Bodin L, Bergstrom G, Lindfors P, Svedberg P. Work-home interference and burnout: a study based on Swedish twins. J Occup Environ Med. 2014;56(4):361-6.

5. Magnusson-Hanson LL, Leineweber C, Chungkham HS, Westerlund $\mathrm{H}$. Work-home interference and its prospective relation to major depression and treatment with antidepressants. Scand J Work Environ Health. 2014;40(1):66-73.

6. Voss M, Josephson M, Stark S, Vaez M, Alexanderson K, Alfredsson L, et al. The influence of household work and of having children on sickness absence among publicly employed women in Sweden. Scand J Public Health. 2008;36(6):564-72.

7. Clays E, Kittel F, Godin I, Bacquer DD, Backer GD. Measures of work-family conflict predict sickness absence from work. J Occup Environ Med. 2009;51(8):879-86.

8. Lidwall U, Marklund S, Voss M. Work-family interference and long-term sickness absence: a longitudinal cohort study. Eur J Public Health. 2010;20(6):676-81.

9. Jansen NW, Kant IJ, van Amelsvoort LG, Kristensen TS, Swaen GM, Nijhuis FJ. Work-family conflict as a risk factor for sickness absence. Occup Environ Med. 2006;63(7):48894.

10. Väänänen A, Kumpulainen R, Kevin MV, Ala-Mursula L, Kouvonen A, Kivimäki M, et al. Work-family characteristics as determinants of sickness absence: a large-scale cohort study of three occupational grades. J Occup Health Psychol. 2008;13(2): $181-96$.

11. Allebeck P, Mastekaasa A. Risk factors for sick leave - general studies. Scand J Public Health. 2004;32(Supplement 63):49-108.

12. Social Insurance Agency. Social insurance in figures 2015 .

13. OECD. Sickness, disability and work: breaking the barriers - a synthesis of findings across OECD countries. Paris: OECD Publishing; 2010.

14. Allen TD, Herst DE, Bruck CS, Sutton M. Consequences associated with work-to-family conflict: a review and agenda for future research. J Occup Health Psychol. 2000;5:278-308.

15. Greenhaus JH, Beutell NJ. Sources of conflict between work and family roles. Acad Manag Rev. 1985;10:76-88.

16. Greenhaus JH, Allen TD, Spector PE. Health consequences of work-family conflict: the dark side of the work-family interface. In: Ganster DC, Perrewe PL, editors. Research in occupational stress and well-being. Amsterdam: JAI Press; 2006. p. 171-211. 
17. Frone MR, Russell M, Cooper ML. Antecedents and outcomes of work-family conflict: testing a model of the work-family interface. $\mathrm{J}$ Appl Psychol. 1992;77:65-78.

18. Amstad FT, Meier LL, Fasel U, Elfering A, Semmer NK. A metaanalysis of work-family conflict and various outcomes with a special emphasis on cross-domain versus matching-domain relations. J Occup Health Psychol. 2011;16:151-69.

19. Demerouti E, Bakker AB, Bulters A. The loss spiral of work pressure, work-home interference and exhaustion: reciprocal relationships in a three-wave study. J Vocat Behav. 2004;64:131-49.

20. Ford M, Heinen B, Langkamer K. Work and family satisfaction and conflict: a meta-analysis of cross-domain relations. J Appl Psychol. 2007;92:57-80.

21. Allen TD, Finkelstein LM. Work-family conflict among members of full-time dual-earner couples: an examination of family life stage, gender, and age. J Occup Health Psychol. 2014;19(3):376-84.

22. Casini A, Clays E, Godin I, De Backer G, Kornitzer M, Kittel F. The differential impact of job isostrain and home-work interference on indicators of physical and mental health in women and men. J Occup Environ Med. 2010;52(12):1236-44.

23. Battams S, Roche AM, Fischer JA, Lee NK, Cameron J, Kostadinov V. Workplace risk factors for anxiety and depression in male-dominated industries: a systematic review. Health Psychology \& Behavioural Medicine. 2014;2(1):983-1008.

24. Mather L, Bergström G, Blom V, Svedberg P. High job demands, job strain, and iso-strain are risk factors for sick leave due to mental disorders. A prospective Swedish twin study with a 5-year followup. J Occup Environ Med. 2015;57(8):858-65.

25. Westerlund H, Gustafsson PE, Theorell T, Janlert U, Hammarström A. Social adversity in adolescence increases the physiological vulnerability to job strain in adulthood: a prospective population-based study. PLoS One. 2012;7(4):e35967.

26. Svedberg P, Ropponen A, Alexanderson K, Lichtenstein P, Narusyte J. Genetic susceptibility to sickness absence is similar among women and men: findings from a Swedish twin cohort. Twin Res Human Genet. 2012;15(05):642-8.

27. Mather L, Bergstrom G, Blom V, Svedberg P. The covariation between burnout and sick leave due to mental disorders is explained by a shared genetic liability: a prospective Swedish twin study with a five-year follow-up. Twin Res Human Genet. 2014;17(6):535-44.

28. Maas H, Spinath FM. Personality and coping with professional demands: a behavioral genetics analysis. J Occup Health Psychol. 2012;17(3):376-85.

29. Bergen SE, Gardner CO, Kendler KS. Age-related changes in heritability of behavioral phenotypes over adolescence and young adulthood: a meta-analysis. Twin Res Human Genet. 2007;10:423-33.
30. McGue M, Bouchard TJJ, Iacono WG, Lykken DT. Behavior genetics of cognitive ability: a life-span perspective. In: Plomin R, McClearn GE, editors. Nature, nurture, and psychology. Washington, DC: American Psychological Association; 1993. p. 59-76.

31. Rantanen J, Kinnunen U, Pulkkinen L, Kokko K. Developmental trajectories of work-family conflict for Finnish workers in midlife. J Occup Health Psychol. 2012;17(3):290-303.

32. Bienvenu OJ, Davydow DS, Kendler KS. Psychiatric 'diseases' versus behavioral disorders and degree of genetic influence. Psychol Med. 2011;41(1):33-40.

33. Torvik FA, Gjerde LC, Røysamb E, Tambs K, Kendler KS, Czajkowski NO, et al. Genetic and environmental contributions to the relationship between internalizing disorders and sick leave granted for mental and somatic disorders. Twin Res Human Genet. 2014;17(4):225-35.

34. Lichtenstein P, Sullivan PF, Cnattingius S, Gatz M, Johansson S, Carlstrom E, et al. The Swedish Twin Registry in the third millennium: an update. Twin Res Human Genet. 2006;9(6):875-82.

35. WHO. International statistical classification of diseases and related health problems, tenth revision (ICD-10). Geneva: WHO; 1993.

36. Dallner M, Elo A-L, Gamberale F, Hottinen V, Knardahl S, Lindström K, et al. Validation of the general nordic questionnaire, QPSNordic, for psychological and social factors at work. Copenhagen: Nordic Council of Ministers; 2000. p. 12

37. Sanne B, Torp S, Mukletun A, Dahl A. The Swedish DemandControl-Support Questionnaire ( DCSQ): factor structure, item analyses, and internal consistency in a large population. Scand J Public Health. 2005;33:166-74.

38. Karasek R, Theorell T. Healthy work: stress, productivity and the reconstruction of working life. New York: Basic Books; 1990.

39. McGue M, Osler M, Christensen K. Causal inference and observational research: the utility of twins. Perspect Psychol Sci. 2010;5: 546-56.

40. van Dongen J, Slagboom PE, Draisma HH, Martin NG, Boomsma DI. The continuing value of twin studies in the omics era. Nat Rev Genet. 2012;13:640-53.

41. RØvik JO, Tyssen R, Hem E, Gude T, Ekeberg Ø, Moum T, et al. Job stress in young physicians with an emphasis on the work-home Interface: a nine-year, nationwide and longitudinal study of its course and predictors. Ind Health. 2007;45(5):662-71.

42. Leijon O, Josephson M, Osterlund N. How common is change of primary diagnosis during an episode of sickness benefit? A register study of medical sickness certificates issued 2010-2012 in Sweden. Scand J Public Health. 2015;43(1):44-51. 